\title{
HYPERTENSION
}

\section{Role of renal ACE in response to hypertensive stimuli}

The importance of the renin-angiotensin system (RAS) in the regulation of blood pressure is undisputed; however, the contribution of tissue-specific RAS to blood-pressure control and the development of hypertension has been a matter of debate. "The finding that RAS blockers effectively reduce blood pressure when most hypertensive patients lack consistent signs of systemic RAS activation is a long-standing clinical conundrum and has prompted many investigators to suggest that alterations in tissue-specific RAS underlie hypertension," explains

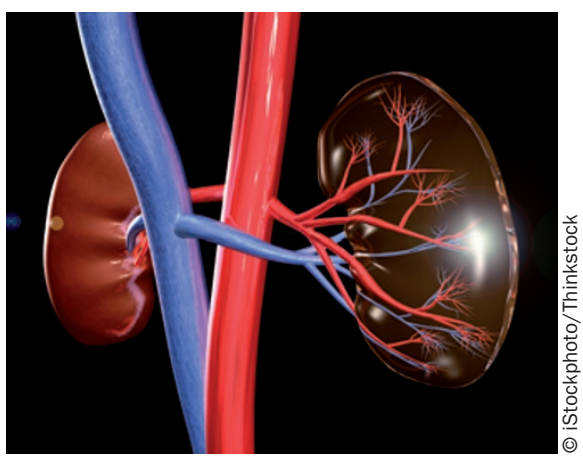

researcher Romer Gonzalez-Villalobos. In a new study, Gonzalez-Villalobos and colleagues used genetic manipulation to show that the absence of kidney angiotensin-converting enzyme (ACE) protects against hypertension, suggesting a key role for renal ACE in the response to hypertensive stimuli.

The researchers used two strains of mice in which homologous recombination was used to direct ACE expression to specific cell types while eliminating the enzyme from the kidneys and other tissues. Both mouse strains had normal levels of circulating ACE and plasma angiotensin (Ang) II. Infusion of Ang II or inhibition of nitric oxide synthesis induced hypertension in wildtype mice but induced only a blunted hypertensive response in both strains lacking kidney ACE.

To further assess the effects of intrarenal Ang II on blood pressure, Gonzalez-Villalobos et al. studied the renal responses of mutant mice to Ang II infusion. Absence of kidney
ACE prevented intrarenal Ang II accumulation, the induction of sodium and water retention and the activation of several renal ion transporters. "We found that even in the presence of high plasma Ang II levels, renal ACE activity is required to increase local Ang II levels in the kidney, which in turn is essential to stimulate sodium transport in the loop of Henle and the distal nephron and induce hypertension," says Gonzalez-Villalobos. "Thus, we have uncovered a very unique and nonredundant role for renal ACE in the setting of hypertension, that is, that the regulation of the sodium excretory pathway is ultimately under the control of the renal RAS and not the systemic RAS, which suggests that the future of RAS blockade might be in organ-specific interventions," he concludes.

Susan J. Allison

Original article Gonzalez-Villalobos, R. A. et al. The absence of intrarenal ACE protects against hypertension. J. Clin. Invest. doi:10.1172/JCI65460 